\title{
Simple white organic light emitting diodes with improved color stability and efficiency using phosphorescent and fluorescent emitters
}

\author{
Heume-II Baek and Changhee Lee ${ }^{\text {a) }}$ \\ School of Electrical Engineering and Computer Science, Inter-University Semiconductor Research Center, \\ Seoul National University, Seoul 151-744, Republic of Korea
}

(Received 23 February 2008; accepted 4 April 2008; published online 17 June 2008)

\begin{abstract}
White organic light emitting diodes (WOLEDs) with both phosphorescent and fluorescent emitting layers (EML) usually adopt an interlayer between them to achieve high efficiency by preventing mutual quenching, but insertion of the interlayer causes a higher operating voltage as well as additional fabrication steps. Here, we demonstrate that simple-structure WOLEDs without an interlayer could be achieved using the combination of phosphor-sensitized-fluorescent red and phosphorescent blue EMLs. In addition, the main cause of the color shift with increasing current density was identified, and the color shift of the WOLED was successfully suppressed by properly balancing emission from the red and blue EMLs. Consequently, a maximum external quantum efficiency of $6.2 \%$ (a current efficiency of $14.3 \mathrm{~cd} / \mathrm{A}$ ) and very stable color coordinates of $(0.32 \pm 0.01,0.42 \pm 0.002)$ were achieved. However, the elimination of an interlayer for the combination with a fluorescent blue EML causes about 50\% decrease in the efficiency and a large change in the color coordinates with the driving current density. (C) 2008 American Institute of Physics. [DOI: 10.1063/1.2939573]
\end{abstract}

\section{INTRODUCTION}

White organic light emitting diodes (WOLEDs) are studied because of their application in lightings, ${ }^{1}$ full-color displays, ${ }^{2}$ and sheet backlight units for liquid crystal displays. Various combinations of dopant-doped emitting layers (EMLs) have been used to generate the desired white light. Recently, efficient WOLEDs were reported to comprise the fluorescent blue emitter together with the phosphorescent green and red emitters, ${ }^{3,4}$ and their efficiency was even higher than those of all phosphorescent WOLEDs. ${ }^{5,6}$ The reason for the higher efficiency is the efficient utilization of both singlet and triplet excitons generated from the host material by properly selecting and positioning each dopantdoped layer. ${ }^{3}$ However, an interlayer (nonemitter doped layer) was inserted to separate the phosphorescent and the fluorescent emitting regions, thereby preventing the mutual quenching between the fluorescent blue and the phosphorescent red and green emitters. ${ }^{4}$ This quenching mainly occurs through the nonradiative triplet energy level of the fluorescent blue emitter, which is positioned lower than the radiative triplet energy level of the phosphorescent green and red emitters.

Another combination of the fluorescent and phosphorescent emitter is by using the phosphor-sensitized fluorescence. ${ }^{7}$ The yellow organic light emitting diodes (OLEDs), which has the bis[2-(2'-benzothienyl)pyridinato- $\mathrm{N}, \mathrm{C}^{3^{\prime}}$ (acetylacetonato)iridium(III) doped -dicarbazolebiphenyl (CBP) layer codoped with [2-methyl-6-[2,3,6,7tetrahydro-1H, 5H-benzo[ij] quinolizin-9-yl)ethenyl]-4H-pyran-4-ylidene]propane-dinitrile (DCM2) and fac-tris(2phenylpyridine) iridium $\left(\operatorname{Ir}(\mathrm{ppy})_{3}\right)$, exhibited a maximum ex-

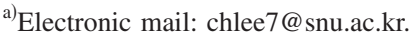

ternal quantum efficiency of $9 \%$ at $0.01 \mathrm{~mA} / \mathrm{cm}^{2,8}$ Therefore, this phosphor-sensitized fluorescence offers a great potential for achieving high efficiency while using a fluorescent emitter. Although several groups reported such WOLEDs, their performances were not so high..$^{9-11}$ To obtain a white light, they combined the phosphor-sensitizedfluorescent yellow EML with the fluorescent blue EML without an interlayer or adjusted each dopant concentration in a single phosphor-sensitized-fluorescent EML. Therefore, their relatively low efficiency might be partly due to improper energy transfer, which could be even severe without an interlayer. More recently, Kanno et al. ${ }^{12}$ replaced the separate phosphorescent red and green EMLs with the single phosphor-sensitized-fluorescent yellow EML in their previous white device structure ${ }^{3}$ and combined it with spatially separated fluorescent blue EML. Their device exhibited a peak external quantum efficiency (EQE) of $8.5 \%$ although they required two interlayers. ${ }^{12}$ However, the insertion of interlayers causes a higher operating voltage as well as additional fabrication steps. Therefore, it is important to design a simpler device structure by reducing the number of interlayers while maintaining the overall device performance as much as possible.

In this study, we demonstrate simple-structure WOLEDs with reasonably high efficiency and good color stability by using the combination of the phosphor-sensitized-fluorescent and phosphorescent EMLs without an interlayer, where CBP codoped with DCM2 and $\operatorname{Ir}(\text { ppy })_{3}$ and CBP doped with iridium(III)bis[(4,6-difluorophenyl)-pyridinato-N,C2']-picolinate (FIrpic) are used as red and blue EMLs, respectively.

\section{EXPERIMENTAL METHODS}

The basic OLED device structure and the energy levels of the materials used in this study are shown in Fig. 1. The 


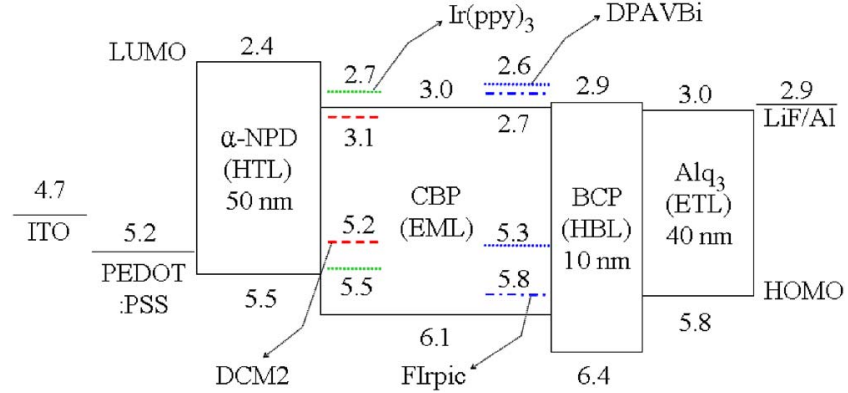

FIG. 1. (Color online) The OLED structures used in this experiment and energy level diagram.

device structure is as follows: indium-tin-oxide (ITO) anode/ poly(3,4-ethylenedioxy-thiophene):poly(styrene sulfonic acid) (PEDOT:PSS, $40 \mathrm{~nm}$ ) hole injection layer/ 4,4'-bis[N-(1-naphthyl)-N-phenyl-amino]biphenyl ( $\alpha$-NPD, $50 \mathrm{~nm}$ ) hole transport layer $(\mathrm{HTL}) /$ various combination of the primary color EMLs/2,9-dimethyl-4, 7 diphenyl-1, 10phenanthroline $(10 \mathrm{~nm})$ hole blocking layer $(\mathrm{HBL}) / \operatorname{tris}(8$ hydroxy-quinoline)-aluminum $\left(\mathrm{Alq}_{3}, 40 \mathrm{~nm}\right)$ electron transport layer $/ \mathrm{LiF}(0.5 \mathrm{~nm}) / \mathrm{Al}(100 \mathrm{~nm})$ cathode. The energy levels of these materials were obtained from the literature. $^{12-14}$

We prepared two sets of devices: one set for the preliminary investigation [(a)-(f) devices] to decide the overall structure of white EMLs, and the other set for the performance comparisons $[(\mathrm{g})-(\mathrm{j})$ devices] among the white light emitting devices with similar structure. All the layers in these devices were prepared by thermal evaporation onto $\mathrm{UV}-\mathrm{O}_{3}$ treated ITO substrates except for the PEDOT:PSS layer, which was prepared by spin-coating at $4000 \mathrm{rpm}$ and drying under vacuum for $30 \mathrm{~min}$. The substrates were ultrasonically cleaned by dipping them into various solvents prior to $\mathrm{UV}_{-} \mathrm{O}_{3}$ treatment. Organic and metal evaporation were conducted under a base pressure of $5 \times 10^{-6}$ Torr without breaking the vacuum, and the evaporation rates were $1-2 \AA / s$ for the organic materials and $4-5 \AA / s$ for the metal. The doping concentration was adjusted by varying the relative evaporation rates of the host and dopant materials, and the evaporation rates were monitored with a quartz-oscillator thickness monitor. The current-voltage $(I-V)$ characteristics were measured by using a Keithley-236 source measurement unit, and the luminance and $\mathrm{EQE}$ were calculated from photocurrent measurement data obtained with a calibrated Si photodiode (Hamamatsu S5227-1010BQ). The electroluminescence spectra were obtained by using a monochromator (Acton ARC275) combined with a photomultiplier detector.

\section{RESULTS AND DISCUSSION}

\section{A. Characteristics of the OLEDs with three-primary- color EMLs}

We fabricated WOLEDs with three-primary-color EMLs, where $5 \mathrm{~nm}$ CBP layer doped with $8 \% \operatorname{Ir}(\text { ppy })_{3}, 8 \mathrm{~nm}$ CBP layer codoped with $0.5 \%$ DCM2 and $8 \% \operatorname{Ir}(\text { ppy })_{3}$, and 6 nm CBP layer doped with $8 \%$ FIrpic were used as the green $(\mathrm{G})$, red (R), and blue (B) EMLs, respectively. The inset of Fig. 2 shows six EML structures [(a)-(f)] in device set I,

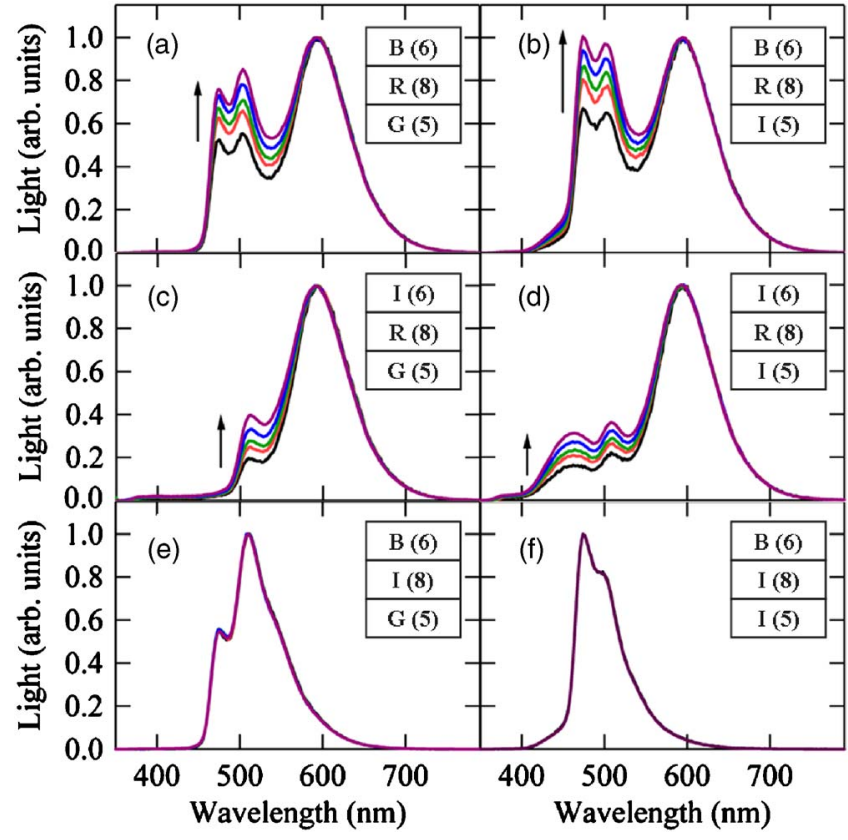

FIG. 2. (Color online) The normalized electroluminescence spectra of device set I. Each inset shows the corresponding EML structure of devices (a)-(f), where bottom and top are continued with HTL and HBL, respectively. The numbers inside the bracket indicate the thickness of each layer in $\mathrm{nm}$ scale. The arrows indicate the spectral variations with increasing current density from 5 to $100 \mathrm{~mA} / \mathrm{cm}^{2}$.

which was prepared in the same batch of evaporation with the combination of various metal masks so that several devices can share the color EML with exactly the same composition. Device (a) was the three-primary-color white OLED and the others were prepared as the control devices. In the EML structures in the inset of Fig. 2, "I" indicates an undoped CBP layer used to maintain the same thickness of EML in each device.

Figure 2 shows the normalized electroluminescence (EL) spectra of devices (a)-(f) at various driving current densities. Each EL spectrum is normalized at the maximum wavelength. Device (a) exhibited three-peak EL spectra: 590, 510, and $470 \mathrm{~nm}$ peaks from DCM2, $\operatorname{Ir}(\mathrm{ppy})_{3}$, and FIrpic emitters, respectively. Device (b) also showed three-peak EL spectra with an additional shoulder at $450 \mathrm{~nm}$ from $\alpha$-NPD HTL due to the existence of the undoped CBP (I) layer. The highest occupied molecular orbital (HOMO) energies of $\alpha$-NPD, $\mathrm{CBP}$, and $\operatorname{Ir}(\mathrm{ppy})_{3}$ are known to be $5.5,6.1$, and $5.5 \mathrm{eV}$, respectively, ${ }^{12-14}$ and hole injection from $\alpha$-NPD into the undoped CBP (I) layer is less efficient than into the $\operatorname{Ir}(\mathrm{ppy})_{3}$-doped CBP layer because a direct hole injection to the $\operatorname{Ir}(\mathrm{ppy})_{3}$ molecule is possible due to the almost the same HOMO energy as that of $\alpha$-NPD. Similarly, devices (d) and (f) also exhibited an additional $450 \mathrm{~nm}$ peak. Interestingly, the spectra of devices (a) and (b) and those of devices (c) and (d) resembled each other when excluding this additional peak from $\alpha$-NPD. The spectra of device (d) also showed a small amount of $\operatorname{Ir}(\mathrm{ppy})_{3}$ emission as already known in previous literature. ${ }^{8}$ This means that almost all excitons in the $\mathrm{G}$ layer transferred to the $\mathrm{R}$ layer possibly due to the higher hole conduction property of the $\operatorname{Ir}(\mathrm{ppy})_{3}$ doped CBP layer ${ }^{15}$ and common use of $\operatorname{Ir}(\mathrm{ppy})_{3}$ in both $\mathrm{R}$ and $\mathrm{G}$ layers. A rela- 


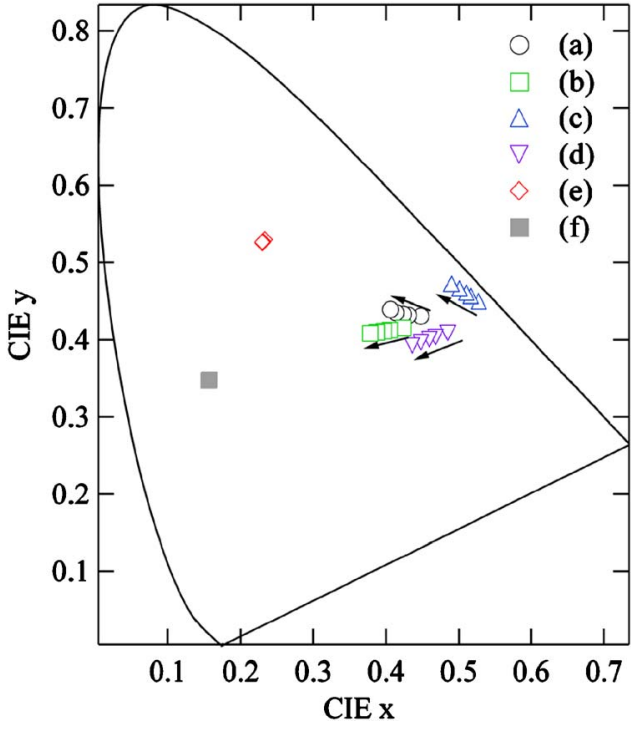

FIG. 3. (Color online) The CIE color coordinates of devices (a)-(f). The arrows indicate the color shift direction with increasing current density from 5 to $100 \mathrm{~mA} / \mathrm{cm}^{2}$.

tively small FIrpic emission was observed in device (e) as compared to other devices with the B layer due to the higher efficiency (nearly 100\% photoluminescence efficiency) of $\operatorname{Ir}(\text { ppy })_{3}$ in the CBP host. ${ }^{16}$

Figure 3 shows the Commission Internationale de L'Eclairage (CIE) color coordinates for devices (a)-(f) at various current densities. Devices (a) and (b) with the threepeak EL spectra exhibited the color coordinates of $(0.406$, $0.439)$ and $(0.378,0.408)$ at $100 \mathrm{~mA} / \mathrm{cm}^{2}$. Therefore, these two devices are not good white devices because their color coordinates are too far from the ideal white point of $(0.33$, 0.33). There were nearly no variation in the EL spectra of devices (e) and (f), in which only phosphorescent emitters are used, as driving current density changes. However, all other devices exhibited a rather large spectral variation and therefore big color coordinate shift. Devices (a)-(d) commonly exhibited relative intensity increases in the shortwavelength region at higher current density. These spectral variations can be attributed to the $\mathrm{R}$ layer because the device (d) with the I/R/I EML sequence also exhibited the same amount of spectral change and color coordinate shift with similar shift direction and amount. In the $\mathrm{R}$ layer, both DCM2 and $\operatorname{Ir}(\text { ppy })_{3}$ emitters are codoped in the CBP host and a relatively deep hole trap is formed by the DCM2 molecule. This hole trap might be filled at a higher current density, and the Förster energy transfer to a positively ionized DCM2 molecule is not possible. ${ }^{17}$ Therefore, the decrease in the red emission (relative emission increase in short wavelength) at higher current density could be partly attributed to the increased fraction of DCM2 molecules occupied by holes.

Device (a) showed a reasonable peak EQE of $6.0 \%$ at $0.1 \mathrm{~mA} / \mathrm{cm}^{2}$, whereas device (b) exhibited only $4.0 \%$ of peak EQE due to the existence of the I layer right after the HTL. The lower efficiency of device (b) compared with device (a) can be understood since the hole injection from $\alpha$-NPD into the undoped CBP is less efficient than into the

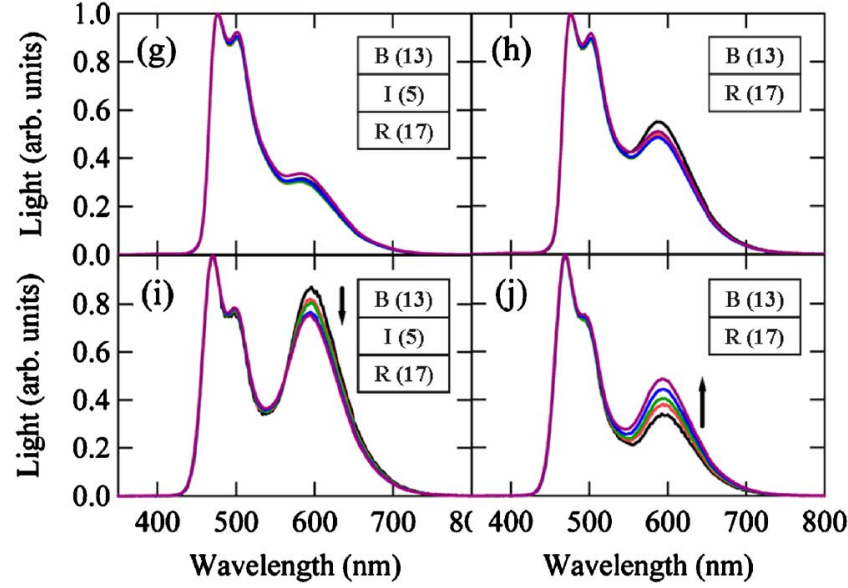

FIG. 4. (Color online) The normalized electroluminescence spectra of device set II. Each inset shows the corresponding EML structure of devices (g)-(j). For the B layer, a FIrpic-doped-CBP layer for devices (g) and (h) and a DPAVBi-doped-CBP layer for devices (i) and (j) were used, respectively. The arrows indicate the spectral variations with increasing current density from 5 to $100 \mathrm{~mA} / \mathrm{cm}^{2}$.

$\operatorname{Ir}(\mathrm{ppy})_{3}$-doped CBP layer, and therefore, some electrons and holes recombine at the $\alpha$-NPD layer [see a shoulder at around $450 \mathrm{~nm}$ in Fig. 2(b)]. The similarity of the EL spectra between devices (a) and (b) and the reasonably high peak EQE of device (a) suggest the possibility of fabricating efficient WOLEDs with only $\mathrm{R}$ and B EMLs.

\section{B. Characteristics of the WOLEDs with two-primary- color EMLs}

The three-peak devices demonstrated in Sec. III A, such as devices (a) and (b), exhibited a very large color shift due to the spectral variations originated from the $\mathrm{R}$ layer (the phosphor-sensitized-fluorescent layer) with increasing current density. In addition, their color coordinates are quite far from the ideal white point. To solve these problems, we increased the portion of the B layer in the overall EML structure so that the spectral change originated from the $\mathrm{R}$ layer could be effectively suppressed and the color coordinate could be moved toward the ideal white point by the increased blue emission intensity.

The inset of Fig. 4 shows four EML structures [from (g) to (j)] in device set II. The thickness of the B EML was increased to $13 \mathrm{~nm}$, compared to the 6-nm-thick B EML in device set I, and the DCM2 concentration was reduced in the $\mathrm{R}$ EML, which consisted of the 17-nm-thick CBP layer codoped with $0.2 \%$ DCM 2 and $8 \% \operatorname{Ir}(\text { ppy })_{3}$. In addition, the overall EML thickness was increased to improve the device efficiency by considering the longer diffusion length of the triplet exciton. Because the main recombination zone is expected to be formed at the EML/HBL interface in our device structure (see Fig. 1), the blue emission could be increased more compared with red emission by this broadened EML, and therefore, a more balanced white emission could be obtained. Two types of B EML were used: $8 \%$ phosphorescent blue dopant (FIrpic)-doped CBP layer for devices (g) and (h), as in Sec. III A, and $4 \%$ fluorescent blue dopant 4 , 4' - bis [2 - $\{4-(\mathrm{N}, \mathrm{N}$-diphenylamino)phenyl $\}$ vinyl $]$ biphenyl $(\mathrm{DPAVBi})^{18}$-doped CBP layer for devices (i) and $(\mathrm{j})$, which 


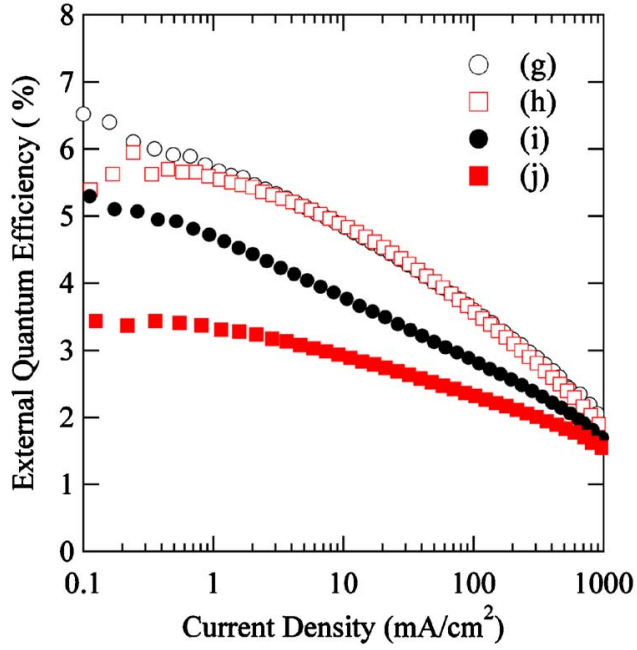

FIG. 5. (Color online) EQEs of the white devices (g)-(j) as a function of the driving current densities.

were prepared for a direct comparison with devices (g) and (h). The DPAVBi concentration of $4 \%$ was chosen since we got a maximum EQE of $\sim 3.2 \%$ at this concentration in our previous experiment (not shown here). In the inset of Fig. 4, "I" indicates a 5-nm-thick undoped CBP layer acting as an interlayer.

Figures 4 and 5 show the normalized EL spectra and $\mathrm{EQE}$ of device set II, respectively. It can be clearly noticed that there was no difference in the EQE for devices (g) and (h) with the phosphorescent blue dopant (FIrpic), while there was a rather large efficiency difference between devices (i) and $(j)$ with the fluorescent blue dopant (DPAVBi). This result indicates that there is no additional loss channel in the EML structure of device (h) even without an interlayer as compared to device (g). The triplet energy level of FIrpic is higher than that of $\operatorname{Ir}(\mathrm{ppy})_{3}$ and the singlet energy level of DCM2. This means that energy transfer from the $\mathrm{B}$ to the $\mathrm{R}$ layer could also be utilized as the red (by DCM2) and green (by $\operatorname{Ir}(\mathrm{ppy})_{3}$ ) emissions. A slightly lower red emission of device (g) compared to device $(\mathrm{h})$ could be attributed to the existence of an interlayer which prevents energy transfer from the B EML (FIrpic) to the R EML ( $\operatorname{Ir}(\mathrm{ppy})_{3}$ and, finally, to DCM2.

Device (h) showed the peak EQE and current efficiency of $6.2 \%$ and $14.3 \mathrm{~cd} / \mathrm{A}$, respectively, at $0.1 \mathrm{~mA} / \mathrm{cm}^{2}$ of driving current density. This is a reasonable value when considering the peak EQE of each EML, because $30 \mathrm{~nm}$ CBP doped with $8 \%$ FIrpic exhibited a peak EQE of about $5 \%$ in our previous experiment and $8 \%$ peak $\mathrm{EQE}$ of phosphorsensitized-fluorescent R EML has been reported. ${ }^{8}$

Differently, there was a rather large efficiency difference in the EQE of devices (i) and (j) with the fluorescent blue dopant (DPAVBi). The peak EQEs of devices (i) and (j) were $5.3 \%$ and $3.4 \%$ at $0.1 \mathrm{~mA} / \mathrm{cm}^{2}$, respectively. In addition, the shape of the normalized spectra of devices (i) and (j) exhibited an opposite tendency to that of devices $(\mathrm{g})$ and $(\mathrm{h})$. The spectrum of device (i) showed a higher red emission than that of device $(\mathrm{j})$. This means that a significant portion of triplet excitons, formed in the R EML, transferred to the B

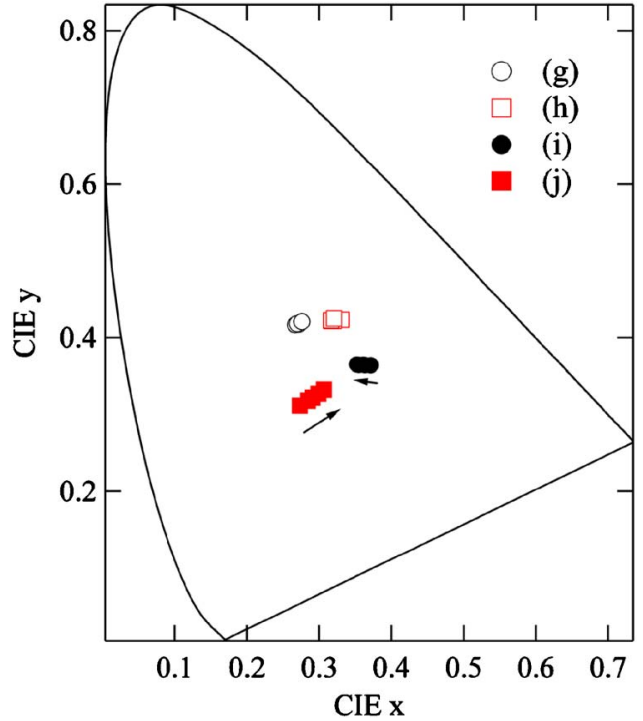

FIG. 6. (Color online) The CIE color coordinates of the white devices (g)-(j). The arrows indicate the color shift direction with increasing current density from 5 to $100 \mathrm{~mA} / \mathrm{cm}^{2}$.

EML and was quenched by the nonradiative triplet energy level of DPAVBi when an interlayer was not used.

Figure 6 shows the variation in the CIE color coordinates of device set II at various current densities. The color coordinates of device set II approached the ideal white point of $(0.33,0.33)$ compared to those of devices (a) and (b) in Sec. III A. In addition, the color coordinate change was very small, especially for devices (g) and (h); (0.32 $\pm 0.01,0.42 \pm 0.002$ ) from 5 to $100 \mathrm{~mA} / \mathrm{cm}^{2}$ of the driving current density range were obtained in device (h). This well suppressed color coordinate shift characteristics can be mainly attributed to the relatively increased blue emission because almost all color shifts with the driving current density change was originated from the phosphor-sensitizedfluorescent EML as already analyzed in Sec. III A. Contrarily, device (j) exhibited the largest color coordinate shift of $(0.29 \pm 0.02,0.32 \pm 0.01)$ over $5-100 \mathrm{~mA} / \mathrm{cm}^{2}$ driving current density range among device set II devices, while that of device (i) with the same B EML was of the same level with those of devices (g) and (h). Therefore, an interlayer is essential to acquire the desired white emission with proper efficiency and small color coordinate shift characteristics in the EML combination with the fluorescent blue dopant. Consequently, we can remove an interlayer in the combination of the phosphor-sensitized-fluorescent red and phosphorescent blue EMLs without sacrificing the overall performance of the WOLEDs.

\section{CONCLUSIONS}

We demonstrated that a simple and efficient WOLED could be achieved with the stack of the phosphor-sensitizedfluorescent and phosphorescent EMLs without using an interlayer between them, where a CBP layer codoped with DCM2 and $\operatorname{Ir}(\mathrm{ppy})_{3}$ and a CBP layer doped with FIrpic were used as the red and blue EMLs, respectively. The same level of efficiency as that of the device with an interlayer in this EML stack was obtained, whereas the elimination of an in- 
terlayer for the combination with a fluorescent blue EML causes about $50 \%$ decrease in the efficiency. In addition, the main source of the color coordinate shift with the driving current density change was identified to be the phosphorsensitized-fluorescent red EML through a spectral comparison among several control devices. Based on this finding, we increased the portion of the blue EML in the overall EML stack, and therefore, good color stability over a broad driving current density range could also be achieved. Consequently, a maximum EQE of $6.2 \%$ (a current efficiency of $14.3 \mathrm{~cd} / \mathrm{A}$ ) at $0.1 \mathrm{~mA} / \mathrm{cm}^{2}$ and very stable color coordinates of $(0.32 \pm 0.01,0.42 \pm 0.002)$ over $5-100 \mathrm{~mA} / \mathrm{cm}^{2}$ were obtained by properly balancing the light emission from the red and blue EMLs.

\section{ACKNOWLEDGMENTS}

This work was supported by Seoul R\&BD support program (CR070048) and Samsung SDI-Seoul National University Display Innovation Program.

${ }^{1}$ B. W. D'Andrade and S. R. Forrest, Adv. Mater. (Weinheim, Ger.) 16, 1585 (2004).

${ }^{2}$ T. K. Hatwar, J. P. Spindler, M. L. Ricks, R. H. Young, Y. Hamada, N. Saito, K. Mameno, R. Nishikawa, H. Takahashi, and G. Rajeswaran, Proc.
SPIE 5214, 233 (2004).

${ }^{3}$ Y. Sun, N. C. Giebink, H. Kanno, B. Ma, M. E. Thompson, and S. R. Forrest, Nature (London) 440, 908 (2006).

${ }^{4}$ G. Schwartz, K. Fehse, M. Pfeiffer, K. Walzer, and K. Leo, Appl. Phys. Lett. 89, 083509 (2006).

${ }^{5}$ B. W. D'Andrade, M. E. Thompson, and S. R. Forrest, Adv. Mater. (Weinheim, Ger.) 14, 148 (2002).

${ }^{6}$ H. Kanno, Y. Sun, and S. R. Forrest, Appl. Phys. Lett. 86, 263502 (2005).

${ }^{7}$ M. A. Baldo, M. E. Thompson, and S. R. Forrest, Nature (London) 403, $750(2000)$

${ }^{8}$ B. W. D'Andrade, M. A. Baldo, C. Adachi, J. Brooks, M. E. Thompson, and S. R. Forrest, Appl. Phys. Lett. 79, 1045 (2001).

${ }^{9}$ G. Cheng, F. Li, Y. Duan, J. Feng, S. Liu, S. Qiu, D. Lin, Y. Ma, and S. T. Lee, Appl. Phys. Lett. 82, 4224 (2003).

${ }^{10}$ G. Lei, L. Wang, and Y. Qiu, Appl. Phys. Lett. 85, 5403 (2004).

${ }^{11}$ Y. Zhang, G. Cheng, Y. Zhao, J. Hou, and S. Liu, Appl. Phys. Lett. 86, 011112 (2005).

${ }^{12}$ H. Kanno, Y. Sun, and S. R. Forrest, Appl. Phys. Lett. 89, 143516 (2006).

${ }^{13}$ Y. Hamada, H. Kanno, T. Tsujioka, H. Takahashi, and T. Usuki, Appl. Phys. Lett. 75, 1682 (1999).

${ }^{14}$ R. J. Holmes, S. R. Forrest, Y. J. Tung, R. C. Kwong, J. J. Brown, S. Garon, and M. E. Thompson, Appl. Phys. Lett. 82, 2422 (2003).

${ }^{15}$ N. Matsusue, S. Ikame, Y. Suzuk, and H. Naito, Appl. Phys. Lett. 85, 4046 (2004).

${ }^{16}$ Y. Kawamura, K. Goushi, J. Brooks, J. Brown, H. Sasabe, and C. Adachi, Appl. Phys. Lett. 86, 071104 (2005)

${ }^{17}$ K. O. Cheon and J. Shinar, Appl. Phys. Lett. 84, 1201 (2004).

${ }^{18}$ Y. H. Ho, T. C. Lim, C. F. Wu, and J. H. Lee, Proc. SPIE 6333, 633303 (2006). 